\title{
Measured Output Voltages of Piezoelectric Devices Depend on the Resistance of Voltmeter
}

\author{
Yewang Su,* Canan Dagdeviren, * and Rui Li
}

Piezoelectric mechanical energy harvester $(\mathrm{MEH})$ has been developed as an important emerging variant of piezoelectric devices. Experiments in the literature show that the voltage-time curves of piezoelectric devices encompass both positive and negative characteristics even though the strain in the piezoelectric material is always positive during the applied cycling load. This does not agree with the results predicted by the piezoelectric theory of open circuit. Here, both the experiments and theory are performed to understand this important problem. A zirconate titanate (PZT) MEH is fabricated and the output voltages are recorded with three voltmeters. It is found that the measured voltages depend on the resistance of voltmeter. The peak value of voltage increases with the increase of the resistance of voltmeter, which is contrary to the established knowledge that the measurement results are independent of the instruments used. A theoretical model considering the voltmeter with finite resistance is established. The charge is allowed to go through the voltmeter and switch the directions during increasing and releasing of strain. The results by this model agree well with those from the experiments. The findings suggest that the resistance of voltmeter should be reported for voltage measurement of the piezoelectric devices.

\section{Introduction}

As being power sources for wearable or implantable electronic devices, piezoelectric materials such as zinc oxide ( $\mathrm{ZnO})$, lead zirconate titanate (PZT), and polyvinylidene fluoride (PVDF) offer potential for converting electrical power from mechanical motion associated with natural body processes (e.g., walking and breathing). ${ }^{[1]}$ The prospective of utilizing piezoelectric materials to generate power from everyday activities and integrating them in devices permits the applications of devices without external power source or battery. Several examples of various materials and device configurations appear in the literature.

An example of piezoelectric materials that has been constantly studied is $\mathrm{ZnO}$, mostly in nanowire (NW) form. ${ }^{[2-4]} \mathrm{A}$ nanogenerator (NG) based on $\mathrm{ZnO} \mathrm{NW}$ was an application that utilizes the piezoelectric effect on NWs to harvest energy from the environment for electricity. ${ }^{[5]} \mathrm{A}$ high-level current of $\approx 500 \mathrm{nA}$ and voltage of $\approx 10 \mathrm{mV}$ were obtained. In order to solve the problems of the electrical output stability and mechanical robustness in the previous design, Yang et al. ${ }^{[6]}$ reported a flexible power generator based on a piezoelectric fine wire that was firmly attached to the metal electrodes at both ends. Repeating stretching and releasing a single wire with a strain of $0.05-0.1 \%$ created an oscillating output voltage of up to $\approx 50 \mathrm{mV}$. Xu et al. ${ }^{[7]}$ reported that a lateral integration of 700 rows of $\mathrm{ZnO} \mathrm{NWs}$ provided promising capability to charge an AA battery as it yielded an output voltage of $1.26 \mathrm{~V}$. ZnO NWs had also been used to develop a high-output nanogenerator (HONG) on plastic substrates that could generate power to light up a commercial light-emitting diode (LED). ${ }^{[8]} \mathrm{Li}$ et al. ${ }^{[9]}$ showed the feasibility of harvesting energy from the pulmonary motions and heartbeat of animals.

PZT-based NGs have also been investigated as they can generate higher power outputs than other semiconductive

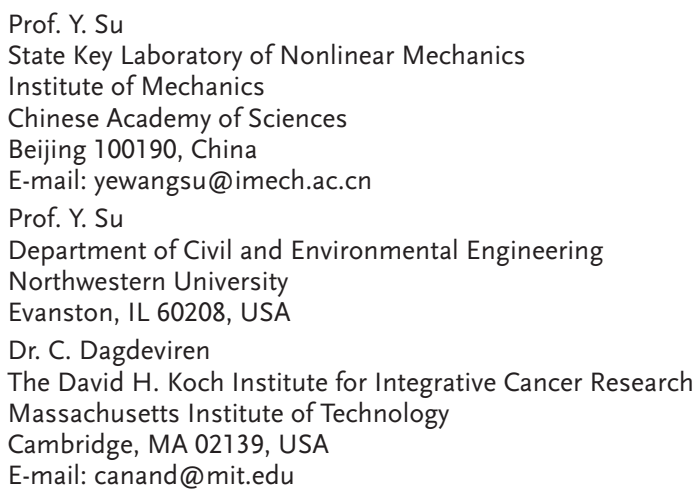

DOI: 10.1002/adfm.201502280

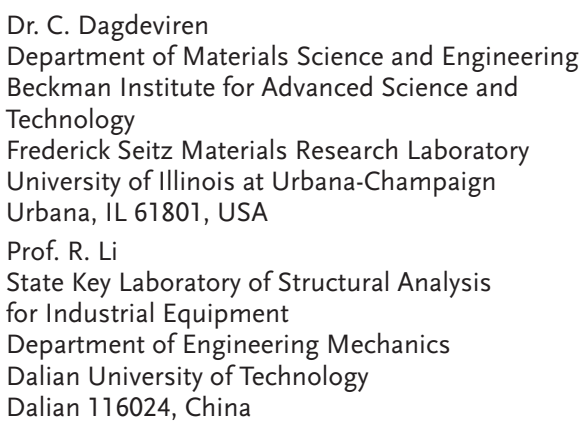

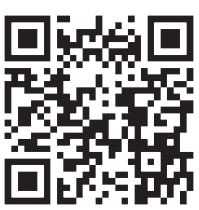

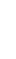


nanomaterials, and can offer high energy conversion efficiency $(\approx 80 \%) .{ }^{[10]}$ Electrospinning process was performed to obtain PZT nanofibers with extremely high piezoelectric voltage constant $\left(\mathrm{g}_{33}, 0.079 \mathrm{Vm} \mathrm{N}^{-1}\right) \cdot{ }^{[1]}$ Despite its excellent piezoelectric property, PZT has a disadvantage due to its brittleness and strain limitation $(<1 \%) .{ }^{[12]}$ To overcome this limitation, devices built with PZT, including other brittle piezoelectric materials, were typically integrated with stretchable substrates, as demonstrated by several research groups. ${ }^{[13-16]}$ For instance, the integration of piezoelectric PZT ribbons onto flexible rubber substrate (PDMS) offered an efficient and flexible energy conversion that opened a new opportunity for future energy harvesting systems. ${ }^{[14,17]}$ In recent years, many emerging piezoelectric devices have been presented with attractive performance. A co-integrated collection of such energy harvesting elements with rectifiers and micro-batteries provided an entire flexible system, being capable of viable integration with the beating heart via medical sutures and operation with a high peak voltage of $8.1 \mathrm{~V}$ and efficiency of $\approx 2 \% .{ }^{[16]}$ Dagdeviren et al. ${ }^{[18]}$ demonstrated composite structures of hard piezoelectric material, and stretchable serpentine (spring-like) conducting metal traces with soft elastomer substrates forming an array of mechanical actuators and sensors. The array is softly and reversibly laminated on the surfaces of nearly any organ system of the human body, for rapid and precise measurement and spatial mapping of viscoelastic properties. Researchers also fabricated the directly written energy harvesters and sensors, which pave the way for a costeffective, high-efficiency manufacturing pathway in wearable, bio-integrated electronics with large deformability. ${ }^{[19,20]}$

The voltage output is an important parameter to determine the performance of piezoelectric devices. The literature shows that the voltage-time curves of piezoelectric devices are characterized by the positive and negative variations (Figure 1a), even though the strain or stress in the piezoelectric material is always positive during the cycling load. ${ }^{[6-9,21-25]}$ The theory of open circuit is usually used to predict the peak of the voltage-time curve, ${ }^{[6-9,22-25]}$ which will be shown and

a

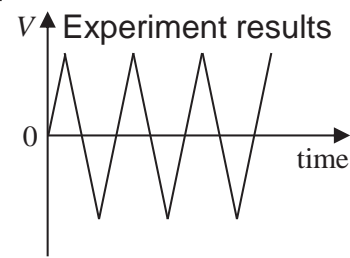

b

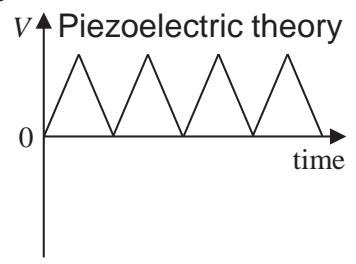

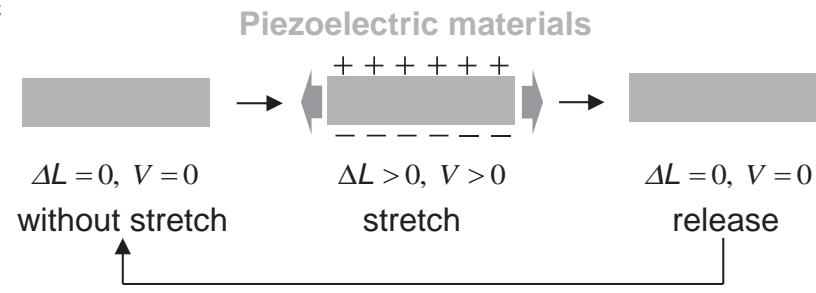

Figure 1. Schematic illustrations of the curves of output voltage versus time from a) experiments and b) piezoelectric theory for open circuit. c) Schematic illustration of the voltage and charge distribution of piezoelectric materials subject to stretching. described analytically in this study. Figure 1c shows the schematic illustration of a piezoelectric ribbon with polarization direction along the thickness direction subject to stretching. By the theory of open circuit, the voltage is zero in the state without being stretched, reaches the maximum while the strain is maximum and restores to zero after being released. The output voltage is always positive during the stretch-release cycles (Figure 1b). The voltage never goes to the range of negative during the process, which conflicts with the experimental findings (Figure 1a). To address this important problem, we both investigated theory model and conducted experimental measurement.

\section{Results}

In order to study the measurement of output voltage, a PZT mechanical energy harvester $(\mathrm{MEH})^{[16]}$ that was flexible enough to wrap a glass tube was fabricated, as shown in Figure S1a (see Supporting Information for the fabrication method details). The functional element of the $\mathrm{MEH}$ is a capacitor-type structure that consists of a layer of PZT (500 nm) between the bottom (Ti/Pt, $20 \mathrm{~nm} / 300 \mathrm{~nm}$ ) and top (Cr/Au, $10 \mathrm{~nm} / 200 \mathrm{~nm}$ ) electrodes (Figure S1b, Supporting Information). A MEH module consists of 12 groups of 10 capacitor structures that are electrically connected in parallel. Each of the twelve groups is connected in series to its neighboring group to increase the output voltage (Figure S1c, Supporting Information).

For the performance characterization of the MEH, a mechanical stage is used to cyclically compress the device from clamps at its ends, and allows doing measurements of output voltage during deformation, as shown in Figure 2. The apparent length of the device is $L=2.5 \mathrm{~cm}$, and the amplitude of the compression $\Delta L$ between the two ends of the device is a periodic function of time $t$. In the experiment, four groups of maximum value of the compression $\Delta L_{\max }=1.5,3,5$, and $10 \mathrm{~mm}$ are performed (Figure 3a), respectively. For each group of maximum compression, three voltmeters with different inner resistance

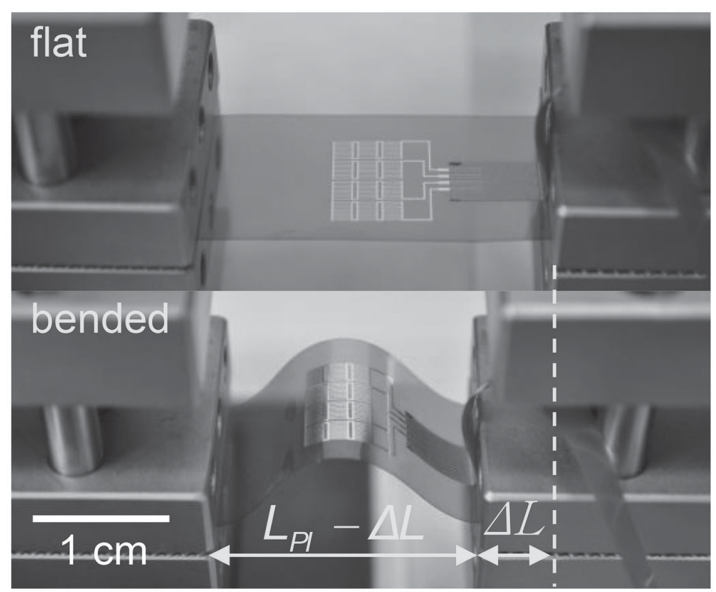

Figure 2. Photographs of a PZT MEH clamped on a bending stage in flat (upper) and bent (lower) configurations. 

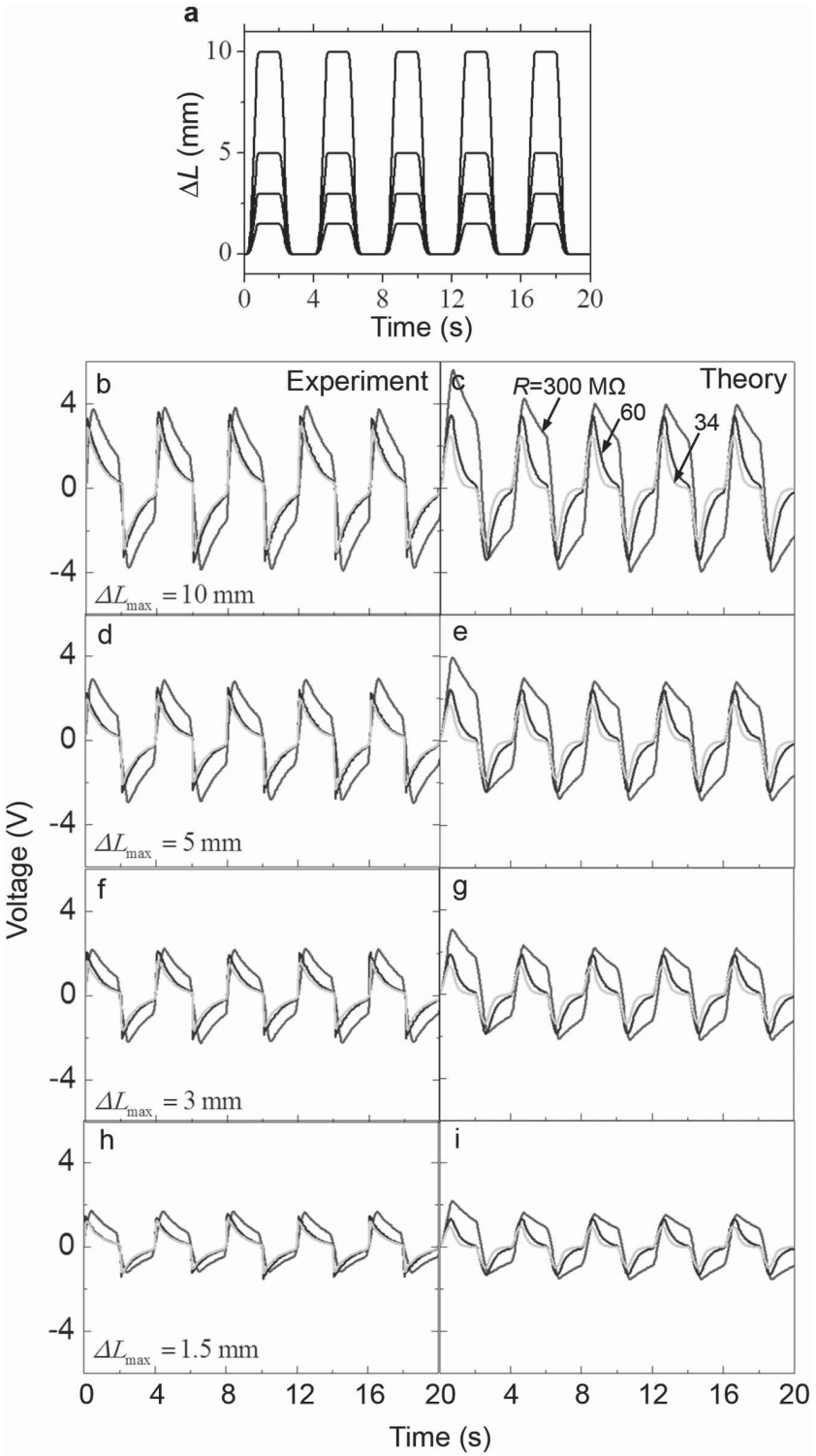

Figure 3. a) Displacement load and the comparison of voltage between $\mathrm{b}, \mathrm{d}, \mathrm{f}, \mathrm{h})$ experiments and $\mathrm{c}, \mathrm{e}, \mathrm{g}, \mathrm{i})$ theory as functions of time for resistance of 34,60 , and $300 \mathrm{M} \Omega$ and maximum displacement of 1.5, 3, 5, and $10 \mathrm{~mm}$.

value $R=34,60$, and $300 \mathrm{M} \Omega$ were used to measure the voltage of the same MEH (see Supporting Information for details). The measured voltage values are different for the same compression $\Delta L_{\max }$, as shown in Figure $3 \mathrm{~b}, \mathrm{~d}, \mathrm{f}$, and $\mathrm{h}$, which is contrary to the established knowledge that the measured results should be independent of the instruments used. The peak value of the voltage output slightly increases with the resistance of the voltmeter (Figure 4). The peak values of output voltage are $2.98,3.61$, and $3.85 \mathrm{~V}$ for the voltmeter with resistances $R=34,60$, and $300 \mathrm{M} \Omega$, respectively, while $\Delta L_{\max }=10 \mathrm{~mm}$ is the same for all cases. The measured results are dependent on not only the device itself, but also the instrument used.

Here, an analytic model coupling the piezoelectric behavior and the finite deformation of the device is developed, as shown

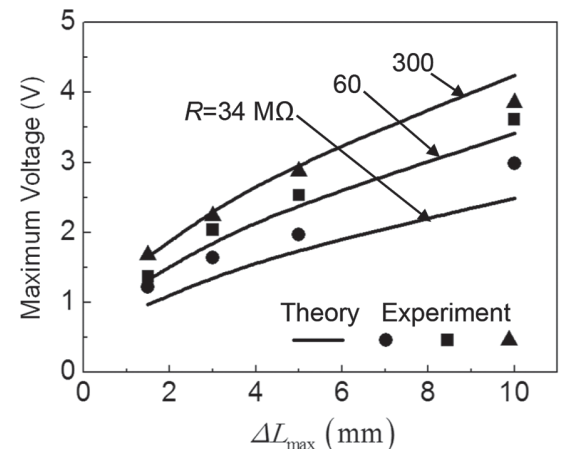

Figure 4. Comparison of the maximum output voltage between theory and experiments for the resistance of 34,60 , and $300 \mathrm{M} \Omega$.

in Figure 5a. When the length of the PZT ribbons is much smaller than that of the supporting substrate (polyimide, PI), the membrane strain in the PZT can be obtained as (see Supporting Information for details)

$\varepsilon_{\mathrm{m}}=4 \pi \frac{\overline{E I}_{\mathrm{PI}}}{\overline{E I}_{\mathrm{comp}}} \frac{h}{L} \sqrt{\frac{\Delta L}{L}}$

where $\overline{E I}_{\text {comp }}$ and $\overline{E I}_{\text {PI }}$ are the bending stiffness (per unit width) of the PI with and without the PZT devices, respectively; $h$ is the distance from the center of the PZT layers to the neutral mechanical plane (Figure S1b, Supporting Information). The capacitor-type structure consists of layers in the following order \{PI, PI, Ti, Pt, PZT, Cr, Au, PI $\}$, as shown in Figure S1b (Supporting Information), where the corresponding moduli are $\left\{\bar{E}_{1 \sim 8}\right\}=\{2.83,2.83,129,196,69.2,292,96.7,2.83\} \mathrm{Gpa}$, and thicknesses $\left\{t_{1 \sim 8}\right\}=\{75,1.2,0.02,0.3,0.5,0.01,0.2,1.2\} \mu \mathrm{m}$. These values yield $\overline{E I}_{\mathrm{PI}} / \overline{E I}_{\text {comp }}=0.45, \gamma_{\text {neutral }}=52.0 \mu \mathrm{m}$ and $h=\left(t_{1}+t_{2}+t_{3}+t_{4}+t_{5} / 2\right)-\gamma_{\text {neutral }}=24.7 \mu \mathrm{m}$.

PZT in this system is transversely isotropic with a polarization direction $x_{3}$ normal to the surface. Constitutive models give relations among the stress $\sigma_{i j}$, strain $\varepsilon_{i j}$, electrical field $E_{i}$ and electrical displacement $D_{i}$ as shown in Equations (S1) and (S2) (Supporting Information), where $c_{i j}$, $e_{i j}$, and $k_{i j}$ are the elastic, piezoelectric, and dielectric constants, respectively. For the plane-strain deformation $\left(\varepsilon_{22}=0\right)$ and traction free on the top surface of the structure $\left(\sigma_{33}=0\right)$, the strain $\varepsilon_{33}$ and electrical field $E_{3}$ along the polarization direction $x_{3}$ satisfy the constitutive relations $0=c_{11} \varepsilon_{11}+c_{13} \varepsilon_{33}-e_{31} E_{3}$ and $D_{3}=e_{31} \varepsilon_{11}+e_{33} \varepsilon_{33}+k_{33} E_{3}$, where the electrical displacement $D_{3}$ along the polarization direction is a constant to be determined. The elimination of $\varepsilon_{33}$ gives $D_{3}=\bar{e} \varepsilon_{11}+\bar{k} E_{3}$, where $\bar{e}=e_{31}-\left(c_{13} / c_{33}\right) e_{33}$ and $\bar{k}=k_{33}+\left(e_{33}^{2} / c_{33}\right)$ are the effective piezoelectric constants. The electrical displacement can be further obtained as

$D_{3}=\bar{e} \varepsilon_{\mathrm{m}}+\frac{\bar{k} V}{N t_{\mathrm{PZT}}}$

from the charge equation $\mathrm{d} D_{3} / \mathrm{d} x_{3}=0$ and the relation $E_{3}=-\partial \phi / \partial x_{3}$ between the electrical field and electrical potential, together with the boundary condition that the voltage difference between the bottom and top of PZT is $V / N$, where $V$ is the total output voltage between the two ends of the $N$ groups 


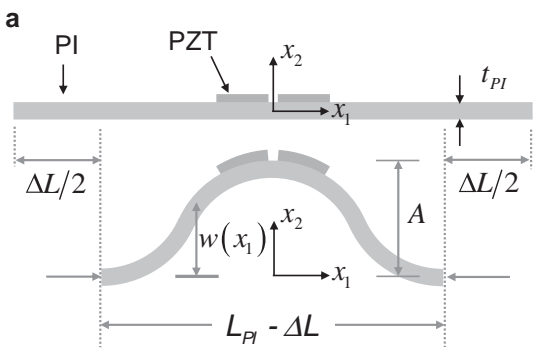

b

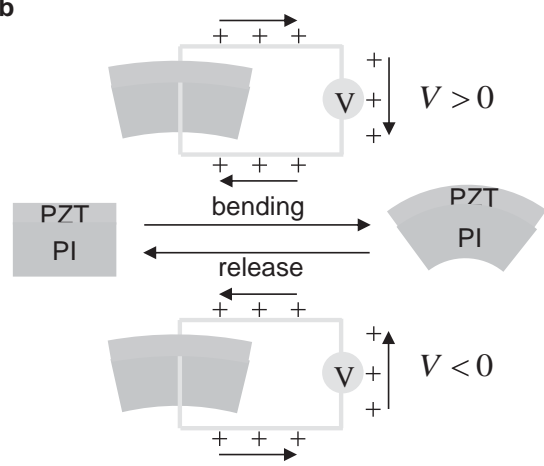

Figure 5. Schematic illustrations of a) theoretical shape for buckling of a PZT MEH under compression and b) charge movement in the circuit during the cycling load.

of PZT ribbons in series, $t_{\mathrm{PZT}}$ and $\varepsilon_{m}$ are the thickness and membrane strain of PZT ribbons.

The output voltages measured with a conventional voltmeter are often recognized as the open-circuit voltage. In this case, the electric displacement $D_{3}$ is zero and the voltage is proportional to the membrane strain, according to Equation (2),

$V=\frac{(-\bar{e}) N t_{\mathrm{PZT}}}{\bar{k}} \varepsilon_{m}$

The results suggest that the sign of the voltage is always same as the membrane strain since $(-\bar{e})>0$. Contrary to this, the reported measurements ${ }^{[6-9,22,23,25]}$ and our experimental results (Figure $1 \mathrm{a}$ and Figure $3 \mathrm{~b}, \mathrm{~d}, \mathrm{f}, \mathrm{h}$ ) show alternating positive and negative values, even for the tests in which the strains/ stresses do not change in sign at any stage of the deformation.

This discrepancy arises from an assumption that the voltmeter has infinite resistance, whereas its actual, finite resistance allows charge to pass, in a way that involves changes in direction as the strain increases and decreases, as shown in Figure $5 \mathrm{~b}$. Here, the current $I=-A_{\mathrm{PZT}} \dot{D}_{3}$ is related to the voltage $V$ and resistance $R$ of the voltmeter by $I=V / R$, where $A_{\mathrm{PZT}}=m\left(w_{\mathrm{PZT}, 1} l_{\mathrm{PZT}, 1}+w_{\mathrm{PZT}, 2} l_{\mathrm{PZT}, 2}\right)$ is the total area of PZT ribbons in each group; $m=10$ is the number of PZT ribbons in each group; $w_{\mathrm{PZT}, 1}=100 \mu \mathrm{m}, w_{\mathrm{PZT}, 2}=140 \mu \mathrm{m}, l_{\mathrm{PZT}, 1}=2.02 \mathrm{~mm}$ and $l_{\mathrm{PZT}, 2}=160 \mu \mathrm{m}$ are the widths and lengths of the two rectangular parts of each PZT ribbon, respectively (Figure S1b, Supporting Information). The result is that $V / R=-A_{\mathrm{PZT}} \dot{D}_{3}$, i.e.,

$\frac{\mathrm{d} V}{\mathrm{~d} t}+\frac{N t_{\mathrm{PZT}}}{A_{\mathrm{PZT}} R \bar{k}} V=-\frac{N \bar{e} t_{\mathrm{PZT}}}{\bar{k}} \frac{\mathrm{d} \varepsilon_{\mathrm{m}}}{\mathrm{d} t}$

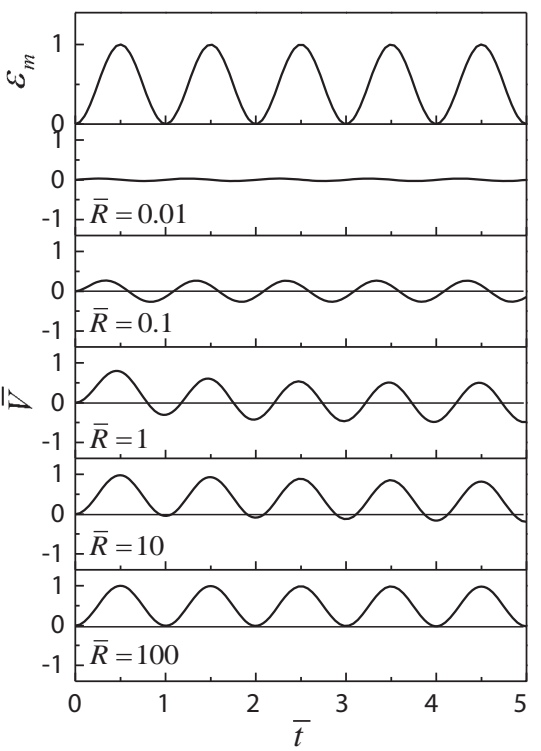

Figure 6. Effects of the dimensionless resistance on the dimensionless voltage.

Using the initial condition $V(t=0)=0$, the voltage is given by

$V=\frac{(-\bar{e}) N t_{\mathrm{PZT}}}{\bar{k}} e^{-\frac{N t_{\mathrm{PZT}}}{A_{\mathrm{PZT}} R \bar{k}}} \int_{0}^{t} \frac{\mathrm{d} \varepsilon_{\mathrm{m}}}{\mathrm{d} t} e^{\frac{N t_{\mathrm{tZT}}}{A_{\mathrm{PZT}} R \bar{k}}} \mathrm{~d} t$

Rewriting this equation with $\bar{V}=\bar{k} V /\left[(-\bar{e}) N t_{\mathrm{PZT}}\right]$ as the dimensionless voltage yields

$\bar{V}=e^{-\overline{\bar{t}}} \int_{0}^{\bar{t}} \frac{\mathrm{d} \varepsilon_{\mathrm{m}}}{\mathrm{d} \bar{t}} e^{\overline{\bar{R}}} \mathrm{~d} \bar{t}$

where $\bar{t}=t / T$ and $\bar{R}=\left[A_{\mathrm{PZT}} \bar{k} /\left(N t_{\mathrm{PZT}} T\right)\right] R$ are the dimensionless time and resistance. For a representative strain $\varepsilon_{m}=[1-\cos (2 \pi \bar{t})] / 2$ as a function of dimensionless time, the dimensionless voltage is

$$
\bar{V}=\frac{\pi \bar{R}}{\sqrt{1+4 \pi^{2} \bar{R}^{2}}} \sin [2 \pi \bar{t}-\arctan (2 \pi \bar{R})]+\frac{2 \pi^{2} \bar{R}^{2}}{1+4 \pi^{2} \bar{R}^{2}} e^{-\bar{t} / \bar{R}}
$$

where the first term of the right side is a periodic function that switches between positive and negative; the second term represents an exponential decay. For infinite resistance, Equation (7) reduces to Equation (3). Figure 6 shows the effect of dimensionless resistance on the dimensionless voltage. The second part of Equation (7) vanishes while $\bar{R}$ is small enough, and the voltage switches between the positive and negative as a periodic function and depends on the resistance. The dimensionless voltage moves to the range of the positive gradually with the increase of the dimensionless resistance. The dimensionless voltage becomes completely positive and is linearly proportional to the membrane strain when the dimensionless resistance is infinitely large, i.e., the open circuit, which is exactly the case of Equation (3). This phenomenon can be understood by the effect of the resistance of the voltmeter. With the increase of 
the resistance value, it becomes more difficult for the charge to go through the voltmeter, and the more stranded charge on electrodes yields higher voltage.

In the experiments, the compression $\Delta L$ between the two ends of the device is a periodic function of time $t$, given by

$$
\Delta L=\left\{\begin{array}{cc}
\frac{\Delta L_{\max }}{4}\left[1-\cos \left(\frac{\pi t}{T_{1}}\right)\right]^{2}, & 0 \leq t<T_{1} \\
\Delta L_{\max }, & T_{1} \leq t<T_{1}+T_{2} \\
\frac{\Delta L_{\max }}{4}\left\{1-\cos \left[\frac{\pi\left(t-2 T_{1}-T_{2}\right)}{T_{1}}\right]\right\}^{2}, & T_{1}+T_{2} \leq t<2 T_{1}+T_{2} \\
0, & 2 T_{1}+T_{2} \leq t<<2\left(T_{1}+T_{2}\right)
\end{array}\right.
$$

in the first period, where $\Delta L_{\max }$ is the maximum compression, and $T=2\left(T_{1}+T_{2}\right)$ is the period. For $T_{1}=0.8 \mathrm{~s}, T_{2}=1.2$ $\mathrm{s}$, and $A_{\mathrm{PZT}}=2.24 \mathrm{~mm}^{2}$ in the experiment, $\bar{e}=-6.2 \mathrm{Cm}^{-2}$ and $\bar{k}=2 \times 10^{-8} \mathrm{C}(\mathrm{Vm})^{-1}$, consistent with values in the literature, ${ }^{[26]}$ Figure $3 c, e, g$,i show the voltage $V$ versus time $t$ obtained from Equation (6), and the peaks are plotted in Figure 6. The theoretical results agree well with the experimental findings, including both peaks and shapes of the voltage-time curves. The peak value of the voltage increases with the resistance of the voltmeter. It confirms that, for piezoelectric devices, the measured output voltages indeed depend on the resistance of the voltmeter. To evaluate the performance of the piezoelectric devices reasonably, the resistance of the voltmeter should be reported in the measurement.

\section{Conclusions}

In summary, output voltage measurement represents a fundamental importance in the performance characterization of emerging classes of flexible and stretchable piezoelectric devices. The literature shows that most of voltage-time curves of piezoelectric devices are characterized by the alternatively positive and negative variations, even though the strain in the piezoelectric material is always positive during the applied cycling load. This phenomenon does not obey the piezoelectric theory for open circuit, which is usually adopted for the quantitative calculation of peak voltages in the literature. Here, we studied this important problem experimentally and theoretically. Collective results show that the measured output voltage depends on the resistance of the voltmeter. The peak of voltage increases with the increase of the resistance of voltmeter. As a significant conclusion, the resistance value of the measurement systems, i.e., voltmeters should be reported in the voltage measurement studies of piezoelectric devices. This conclusion is applicable not only to PZT energy harvester consisting of PZT ribbons, but also to other piezoelectric devices with different configurations, such as sensors and piezoelectric polymers in the form of wire/fibers. ${ }^{[27,28]}$ The established model can be also extended to those piezoelectric devices by reanalyzing the mechanical deformation and piezoelectric behavior. It should be pointed out that the effect described here does not exist for current measurement because the inner resistance of the ampere meter is usually very small or even zero. Our ongoing work based on the findings here is to develop a new voltmeter with infinite or approximately infinite inner resistance for precise characterization of the properties of piezoelectric devices.

\section{Supporting Information}

Supporting Information is available from the Wiley Online Library or from the author.

\section{Acknowledgements}

The authors thank Prof. John A. Rogers of the University of Illinois for his helpful discussions and continued support. C.D. thanks P. Joe for her assistance on experimental part. This work was supported by the National Natural Science Foundation of China (grant 11302038) and Fundamental Research Funds for the Central Universities of China (grant DUT15LK14).

Received: June 4, 2015

Revised: June 25, 2015

Published online: July 27, 2015

[1] T. Starner, IBM Syst. J. 1996, 35, 618.

[2] C. Dagdeviren, S.-W. Hwang, Y. Su, S. Kim, H. Cheng, O. Gur, R. Haney, F. G. Omenetto, Y. Huang, J. A. Rogers, Small 2013, 9, 3398.

[3] C. Dagdeviren, M. Papila, Polym. Compos. 2010, 31, 1003.

[4] S. Xu, Y. Wei, J. Liu, R. Yang, Z. L. Wang, Nano Lett. 2008, 8, 4027.

[5] J. Liu, P. Fei, J. Zhou, R. Tummala, Z. L. Wang, Appl. Phys. Lett. 2008, 92, 173105.

[6] R. Yang, Y. Qin, L. Dai, Z. L. Wang, Nat. Nanotechnol. 2009, 4, 34.

[7] S. Xu, Y. Qin, C. Xu, Y. Wei, R. Yang, Z. L. Wang, Nat. Nanotechnol. 2010, 5, 366.

[8] G. Zhu, R. Yang, S. Wang, Z. L. Wang, Nano Lett. 2010, 10, 3151.

[9] Z. Li, G. Zhu, R. Yang, A. C. Wang, Z. L. Wang, Adv. Mater. 2010, 22, 2534.

[10] A. M. Flynn, S. R. Sanders, IEEE Trans. Power Electron. 2002, $17,8$.

[11] X. Chen, S. Xu, N. Yao, Y. Shi, Nano Lett. 2010, 10, 2133.

[12] T. Tanimoto, K. Okazaki, K. Yamamoto, Jpn. J. Appl. Phys., Part 1 1993, 32, 4233.

[13] E. M. Alkoy, C. Dagdeviren, M. Papila, J. Am. Ceram. Soc. 2009, 92, 2566.

[14] X. Feng, B. D. Yang, Y. Liu, Y. Wang, C. Dagdeviren, Z. Liu, A. Carlson, J. Li, Y. Huang, J. A. Rogers, ACS Nano 2011, 5, 3326.

[15] Y. Qi, J. Kim, T. D. Nguyen, B. Lisko, P. K. Purohit, M. C. McAlpine, Nano Lett. 2011, 11, 1331.

[16] C. Dagdeviren, B. D. Yang, Y. Su, P. I. Tran, P. Joe, E. Anderson, J. Xia, V. Doraiswamy, B. Dehdashti, X. Feng, B. Lu, R. Poston, Z. Khapley, R. Ghaffari, Y. Huang, M. J. Slepian, J. A. Rogers, Proc. Natl. Acad. Sci. U.S.A. 2014, 111, 1927.

[17] Y. Qi, N. T. Jafferis, K. Lyons Jr., C. M. Lee, H. Ahmad, M. C. McAlpine, Nano Lett. 2010, 10, 524.

[18] C. Dagdeviren, Y. Shi, P. Joe, R. Ghaffari, G. Balooch, K. Usgaonkar, O. Gur, P. L. Tran, J. R. Cosby, M. Meyer, Y. Su, R. C. Webb, A. S. Tedesco, M. J. Slepian, Y. Huang, J. A. Rogers, Nat. Mater. 2015, 14728 . 
[19] Y. Duan, Y. Huang, Z. Yin, N. Bu, W. Dong, Nanoscale 2014, 6, 3289.

[20] Y. Ding, Y. Duan, Y. Huang, Energy Technol. 2015, 3, 351.

[21] L. Persano, C. Dagdeviren, Y. W. Su, Y. H. Zhang, S. Girardo,

D. Pisignano, Y. Huang, J. A. Rogers, Nat. Commun. 2013, 4, 1633.

[22] Y. Qi, M. C. McAlpine, Energy Environ. Sci. 2010, 3, 1275.

[23] K.-I. Park, S. Xu, Y. Liu, G.-T. Hwang, S.-J. L. Kang, Z. L. Wang, K. J. Lee, Nano Lett. 2010, 10, 4939.
[24] Y. Yang, Y. Zhou, J. M. Wu, Z. L. Wang, ACS Nano 2012, 6, 8456.

[25] S. Xu, B. J. Hansen, Z. L. Wang, Nat. Commun. 2010, 1, 93.

[26] S. B. Park, C. T. Sun, Int. J. Fracture 1995, 70, 203.

[27] J. Fang, H. Niu, H. Wang, X. Wang, T. Lin, Energy Environ. Sci. 2013, 6, 2196.

[28] L. Persano, C. Dagdeviren, C. Maruccio, L. De Lorenzis, D. Pisignano, Adv. Mater. 2014, 26, 7574. 